\title{
The lost sunspot cycle: Reanalysis of sunspot statistics
}

\author{
I. G. Usoskin ${ }^{1}$, K. Mursula ${ }^{2}$, and G. A. Kovaltsov ${ }^{3}$ \\ 1 Sodankylä Geophysical Observatory (Oulu unit), 90014 University of Oulu, Finland \\ 2 Department of Physical Sciences, 90014 University of Oulu, Finland \\ e-mail: kalevi.mursula@oulu.fi \\ ${ }^{3}$ Ioffe Physical-Techical Institute, 194021 St. Petersburg, Russia \\ e-mail: gena.kovaltsov@pop.ioffe.rssi.ru
}

Received 28 January 2003 / Accepted 11 March 2003

\begin{abstract}
We have recently suggested that one low sunspot cycle was possibly lost in 1790s (Usoskin et al. 2001, A\&A, 370, L31). In this paper we present the results of a rigorous statistical analysis of all available sunspot observations around the suggested additional cycle minimum in 1792-1793. First we estimate the uncertainty of a monthly mean sunspot number reconstructed from a single daily observation. Then we compare, using quantitative statistical tests, the average level of sunspot activity in 1792-1793 with the average activity during the minimum, mid-declining and maximum phases of cycles in the well-measured reference period 1850-1996. We show that, contrary to the results by Krivova et al. (2002), the level of sunspot activity in 1792-1793 is statistically similar to that in the minimum phase, and significantly different from that in the middeclining and maximum phases. Using the estimated uncertainties, we also calculate new, weighted annual values of $R_{\mathrm{g}}$ in 1790-1796 which show a clear minimum in 1792-1793 and a maximum in 1794-1795, supporting the idea of an additional weak cycle in 1790 's.
\end{abstract}

Key words. Sun: activity - Sun: sunspots - Sun: solar-terrestrial relations

\section{Introduction}

We have recently suggested (Usoskin et al. 2001 - to be denoted here U01) that one sunspot cycle was likely missed in 1790s. Recently, a paper (Krivova et al. 2002 - to be denoted K02) has been published where the authors criticize this idea claiming, e.g., that an additional sunspot minimum did not exist in 1792-1793. Unfortunately, the statistical analysis performed in $\mathrm{K} 02$ is not validated by quantitative tests and even contains several errors. In this paper we reanalyze the arguments by K02 about the sunspot minimum in 1792-1793. We use quantitative statistical tests to show that the approach suggested by K02 yields, when performed correctly, that the available record of sunspot observations in 1790s does not exclude but rather supports the possibility for an additional minimum in 1792-1793. We also discuss the other, more indirect arguments presented in K02 against the lost cycle. In another recent paper (Usoskin et al. 2002a - to be denoted U02) we have demonstrated that the idea of the lost cycle is supported by two independent series of auroral observations, and that another solar activity proxy, the cosmogenic radionuclides $\left({ }^{14} \mathrm{C}\right.$ and $\left.{ }^{10} \mathrm{Be}\right)$ do not exclude the possible existence of the additional cycle.

We would like to note that we analyze here the group sunspot numbers (GSN, denoted as $R_{\mathrm{g}}$; see Fig. 1) provided

Send offprint requests to: I. Usoskin,

e-mail: ilya.usoskin@oulu.fi by Hoyt \& Schatten (1998) and not the more traditional Wolf sunspot numbers (WSN), since the GSN series contains the original (not interpolated) sunspot observations. In the next section we study the question how a single daily observation can represent the monthly mean of sunspot activity. Section 3 is devoted to a thorough quantitative analysis of sunspot observations and their statistics during the period under investigation. In Sect. 4 we comment on some indirect arguments presented by $\mathrm{K} 02$ and in Sect. 5 we give our main conclusions.

\section{How a single daily observation can represent the monthly mean?}

The observations made in 1792-1793 according to known records (Hoyt \& Schatten 1998) are mainly isolated daily observations by single observers. There are in total only $20 \mathrm{ob}-$ servations on 16 days during 1792-1793 (see, e.g., Table 2 in U01). Moreover, the observations were not distributed uniformly over this time, with 12 of them forming a period of 8 consecutive days in August-September 1793. The other 8 observations, each on a different day, are quite randomly spread over the period so that they all fall on different months. Moreover, there are no two consecutive months with sunspot observations, except for August-September 1793.

The K02 paper claims that sunspot numbers in 1792-1793 are typical for the mid-declining phase of a sunspot cycle and, 


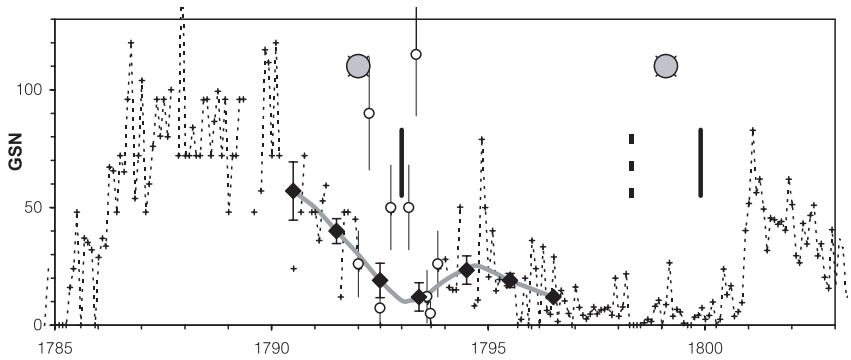

Fig. 1. The monthly group sunspot numbers (Hoyt \& Schatten 1998) outside the years 1792-1793 are shown by the dashed curve. Open dots with error bars depict the estimated monthly means and their standard errors in 1792-1793 (Table 1). The solid diamonds present the estimated weighted annual averages in 1790-1796 (Table 2), and the grey curve gives the spline fit to them. Big grey dots denote the times of the two naked-eye sunspot observations during the depicted interval (Yau \& Stephenson 1988). Vertical solid bars indicate the times of sunspot minima suggested by U01, while the vertical dotted bar denotes the official minimum of cycle 4 based on WSN series.

therefore, exclude the possibility of an additional minimum at this time. They assumed implicitly that one isolated daily observation $R_{\mathrm{d}}$ adequately represents the corresponding monthly mean $R_{\mathrm{m}}=R_{\mathrm{d}}$. However, Hoyt \& Schatten (1998) noted that at least 3 or 4 widely separated days within a month are needed to form a more or less reliable monthly mean. Otherwise, they leave it up to the user of the GSN series to take care of evaluating the usefulness of means of those months that are less covered. This advice was apparently not taken into account by $\mathrm{K} 02$ in their analysis.

Here we examine the question of a correct way to form monthly means $R_{\mathrm{m}}$ from isolated daily observations $R_{\mathrm{d}}$. In order to do that, we analyzed all daily group sunspot numbers for the period 1850-1996 when the data are reliable and contain no observational gaps. We call this data set (more than 53000 daily values) the reference population, assuming that the statistical features of sunspot activity were the same in 1792-1793 and during the reference period.

First, given one of the isolated daily sunspot values $R_{\mathrm{d}}$ observed in 1792-1793, we selected from the reference data set all the days with a daily value close to $R_{\mathrm{d}}$. Then we collected the actual monthly means $R_{\mathrm{m}}$ corresponding to these selected days. (If more than one appropriate daily value are found within a month, the corresponding $R_{\mathrm{m}}$ value is counted as many times). E.g., the highest daily observation $R_{\mathrm{d}}=123$ by Huber in May, 1793, was compared with daily values from the interval [120-130] in the reference data set. (The widths of the bins for the daily values were chosen so as to have sufficient statistics but still remaining within $\pm 10 \%$ of $R_{\mathrm{d}}$.) The corresponding 1200 monthly values (for 450 months) ranged from 52 to 213 with the mean being 115 (see Table 1). If more than one daily observation was done in a month (as, e.g., in August and September 1793) we still can apply the above procedure by looking for the corresponding set of $R_{\mathrm{d}}$ values within the months of the reference period. For the consecutive days of zero value in September 1793 we collected the months with at least five consecutive spotless days.
Table 1. Estimated monthly means $R_{\mathrm{m}}$, their errors $\sigma_{\mathrm{m}}$ and relative weights $w_{\mathrm{m}}$ corresponding to daily sunspot observations $R_{\mathrm{d}}$ in $1792-$ 1793. The best-fit Poisson distribution parameter $\mu$ is also shown. The 2-year mean has been calculated as a weighted average.

\begin{tabular}{cccccc}
\hline \hline month & $R_{\mathrm{d}}$ & $R_{\mathrm{m}}$ & $\sigma_{\mathrm{m}}$ & $w_{\mathrm{m}}$ & $\mu$ \\
\hline Jan. 92 & 24 & 26 & 14 & 0.0051 & 1.7 \\
Apr. 92 & 96 & 90 & 24 & 0.0017 & 7 \\
Jul. 92 & 0 & 7.3 & 7.7 & 0.0169 & 0.9 \\
Oct. 92 & 48 & 50 & 18 & 0.0031 & 3.6 \\
Mar. 93 & 48 & 50 & 18 & 0.0031 & 3.6 \\
May 93 & 123 & 115 & 26 & 0.0015 & 9 \\
Aug. 93 & $24,15,0,0$ & 21.5 & 16 & 0.004 & 1.8 \\
Sep. 93 & $5 * 0$ & 5 & 5 & 0.04 & 0.13 \\
Nov. 93 & 24 & 26 & 14 & 0.0051 & 1.7 \\
1792-1793 & & 16.2 & 7.6 & & \\
\hline
\end{tabular}

From the collected monthly values from the reference population for each month in 1792-1793 with sunspot observations we calculated the means $R_{\mathrm{m}}$ and their errors $\sigma_{\mathrm{m}}$ (see Table 1). These are also shown in Fig. 1 as open dots with error bars. Figure 2 shows samples of histograms of the collected $R_{\mathrm{m}}$ values. The histogram distributions are apparently not Gaussian but can be transformed to the Poisson form after scaling the $\mathrm{X}$-axis. Since a group sunspot number $R_{\mathrm{g}}$ is the number of sunspot groups $G$ multiplied by a factor of 12.08 (Hoyt \& Schatten 1998), the real statistics behind GSN is the statistics of sunspot groups (rather than sunspot numbers) which have much smaller values. Therefore, if $R_{\mathrm{g}}$ is reduced to $G$ by dividing by a factor $k=12$, the statistics of $G=R_{\mathrm{g}} / k$ follow the Poisson distribution:

$f(G, \mu) \propto \frac{\mu^{G} \mathrm{e}^{-\mu}}{G !}$,

where $G$ is an integer $0,1,2, \ldots$ and $\mu$ is the mathematical expectation of the mean. Values of $\mu$ are given in the Col. 6 of Table 1 for all months containing sunspot observations during 1792-1793. Figure 2 shows the best fit Poisson distributions after rescaling of $G$ back to $R_{\mathrm{g}}$. Thus, one can reconstruct a reliable monthly mean $R_{\mathrm{m}}$ (see Table 1 ) even from a single daily observation $R_{\mathrm{d}}$ but its uncertainty is an important factor and should be taken into account.

\section{Sunspot observations in 1792-1793: Statistical analysis}

\subsection{The average sunspot activity}

It was an interesting idea by $\mathrm{K} 02$ to calculate the average sunspot level for $1792-1793$ (denoted as $\overline{R_{92-93}}$ ), i.e., the time around the suggested minimum, and to compare it to the level of some later, better covered solar cycles. However, it is not correct to calculate $\overline{R_{92-93}}$ as a simple arithmetic average of monthly means $R_{\mathrm{m}}$ (as done by K02) since they are of greatly unequal accuracy, as discussed in Sect. 2. (Calculating the $\overline{R_{92-93}}$ mean from daily values is not correct either because daily values are not independent in Aug.-Sep. 1793, as required by the standard averaging methods). In such a case the $\overline{R_{92-93}}$ mean must be calculated as a weighted average. Details of this 

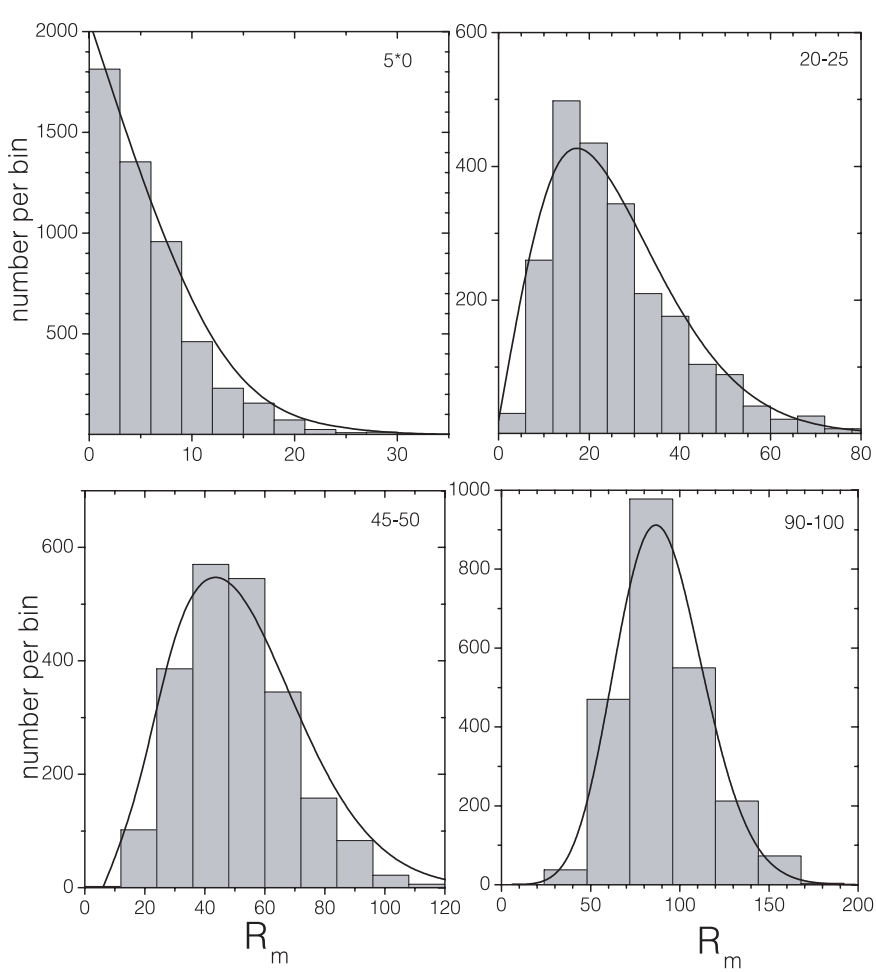

Fig. 2. Samples of histogram distributions of monthly $R_{\mathrm{m}}$ together with the rescaled best-fit Poisson distribution functions. The four panels depict the cases of at least five consecutive zero daily $R_{\mathrm{d}}$ values within one month (marked as $5^{*} 0$ ), and at least one $R_{\mathrm{d}}$ within the interval [20-25], [45-50] or [90-100], respectively.

standard averaging method are given in Appendix. The weights $w_{\mathrm{m}}=1 / \sigma_{\mathrm{m}}^{2}$ used for the averaging are also given in Table 1 . Our final estimate of $\overline{R_{92-93}}$ is $16.2 \pm 7.6$, which is less than half of the value 41 given by K02 (no error estimated there). We note that $\sigma_{\xi}^{*}>\sigma_{\xi}$ (see Appendix) in this case and the factor $l$ (Eq. (A.6)) is about 7, implying that the sample series is inhomogeneous and contains a large systematic error. If the suspicious observation by Huber $\left(R_{\mathrm{g}}=123\right)$ is discarded as suggested by $\mathrm{K} 02$ then $\overline{R_{92-93}}=14.3 \pm 6.0$, cf. the value 31 given by K02. Accordingly, K02 heavily overestimated the average sunspot level in 1792-1793 by using a simple arithmetic average of the monthly values, i.e. taking them with equal weights.

Using the monthly means and their errors, we also calculated the weighted annual $R_{\mathrm{g}}$ values and their errors for the years 1790-1796 (see Table 2). Note that if there are more than 4 independent (i.e., sufficiently widely distributed) observation days within a month, the mean and the standard error of $R_{\mathrm{m}}$ can be calculated directly from the available daily values $R_{\mathrm{d}}$ without employing histograms similar to Fig. 2 . The time profile of the annual $R_{\mathrm{g}}$ values depicted by diamonds in Fig. 1 clearly suggests for an additional minimum at the turn of 1792-1793 and a maximum in 1794-1795.

Following K02, we now estimate in which phase of the solar cycle the sunspot activity level is statistically similar to $\overline{R_{92-93}}$. We have plotted the obtained $\overline{R_{92-93}}$ together with the running 2-year mean of sunspots (grey curve) for the reference period 1850-1996 in Fig. 3a. In the same figure, we have also
Table 2. The formal (Hoyt \& Schatten 1998) and weighted annual averages of $R_{\mathrm{g}}$ and their errors in 1790's.

\begin{tabular}{cccc}
\hline \hline year & formal & weighted & $\sigma$ \\
\hline 1790 & 61.5 & 57 & 12.5 \\
1791 & 43.2 & 39.5 & 5.3 \\
1792 & 42 & 19.2 & 7.3 \\
1793 & 41 & 12.4 & 5.3 \\
1794 & 30.2 & 23.4 & 5.2 \\
1795 & 15.7 & 18.8 & 3 \\
1796 & 13.7 & 12.9 & 3.8 \\
\hline
\end{tabular}

included the running 2-year sunspot activity level obtained by the filtering method of K02, i.e., by selecting for each 730-day interval only those 16 daily $R_{\mathrm{d}}$ values that are separated in time in the same way as the observations in 1792-1793. This filter gives the value $R_{\mathrm{f}}=25.1$ for $1792-1793$ (see Fig. 3). (Note that the horizontal lines in Fig. 3 of K02 present the unweighted averages in 1792-93, not the filter values, contrary to figure caption.) Figure 3 a shows that the obtained value of $\overline{R_{92-93}}=16.2 \pm 7.6$ corresponds very well to the values of $R_{\mathrm{g}}$ around solar minima rather than to the mid-declining phase, contrary to K02. We will test this quantitatively in Sect. 3.3.

\subsection{Probability tests for daily values}

The most systematic part of sunspot observations in 1792-1793 was from August 30 until September 4, 1793. No sunspots were observed during this period. Moreover, there were three independent observers on one day, all reporting no sunspots (Hoyt \& Schatten 1998). K02 correctly listed some examples when several consecutive spotless days can be found even in the middle of declining phase of a cycle. However, noting that this the only period of consecutive observations in 1793, we must calculate how probable it is to find a 6-day spotless period, taking the period randomly within a year. Using the reference population, we have calculated this probability $P(6 * 0)$. Starting from the first day of each year, we slided the 6-day window through one year of data with 1-day time step, counting the number $N$ of times when this window finds only zeroes. (The last window includes 5 days of the next year). Then the probability is defined as

$P(6 * 0)=N / 365$.

The time series of $P(6 * 0)$ is shown in the lower panel of Fig. 3. One can see that the probability peaks within a few years around cycle minima and is zero in all other years. Note also the low probability to find a spotless 6-day period during the recent high activity cycles, even around minima. In addition to this spotless period, daily sunspot values of $R_{\mathrm{d}}=15,24,48$ and 123 were reported in 1793 . We have also calculated the combined probability $P(6 * 0) * P(>R)$ to find a spotless period and at least one day with $R_{\mathrm{d}}$ above a given value $R$ at any time of the year. The probabilities for $R=23$ and $R=45$ are also given in the lower panel of Fig. 3. The probability is still quite high for $P(6 * 0) * P(>23)$, but decreases greatly for $P(6 * 0) * P(>45)$. However, the combined probability to find a spotless 6-day period and at least one daily observation 


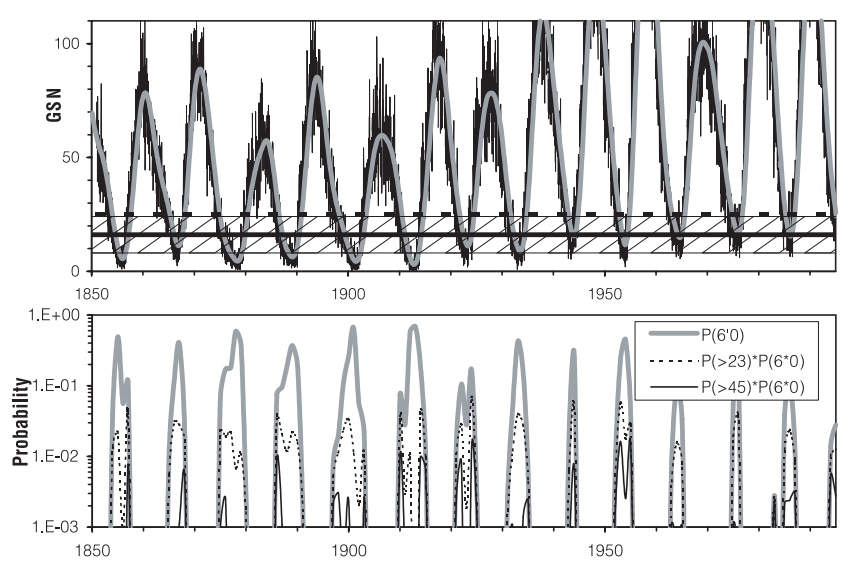

Fig. 3. Top panel: filtered (thin noisy curve) and the 2-year smoothed (grey curve) group sunspot numbers. The thick solid horizontal line and hatched area around it correspond to the weighted sunspot average in $1792-1793 \overline{R_{92-93}}=16.2 \pm 7.6$. The dotted horizontal line denotes the filtered sunspot value $R_{\mathrm{f}}=25.1$ in 1792-1793. Bottom panel: probability to find a spotless 6-day period within one year (grey curve $P(6 * 0)$ ); combined probability to find both a spotless 6 -day period and a day with $R_{\mathrm{g}}>23$ or $R_{\mathrm{g}}>45$ within one year (dotted $P(6 * 0) * P(>23)$ or solid $P(6 * 0) * P(>45)$ lines, respectively).

with $R_{\mathrm{d}}>100$ gets non-zero values only in few years, with the maximum probability $P(6 * 0) * P(>100)$ of about 0.0033 in 1955. Therefore, it is highly improbable to find both a spotless 6-day period and a day with $R_{\mathrm{d}}>100$ when they are taken randomly within one year. This inconsistency gives further motivation for the concern about the correctness of the very high sunspot value $R_{\mathrm{g}}=123$ observed by Huber in May 1793. Let us remind (see U01) that the high $R_{\mathrm{g}}$ corresponding to this observation is due to the very large individual correction factor of Huber (2.564) which is within the 5 largest factors among the 463 observers whose data were included in the GSN series (Hoyt \& Schatten 1998). Therefore, the four sunspot groups reported by Huber on 28 May, 1793, would yield $R_{\mathrm{g}}=48$ but the large correction factor leads to the much higher GSN value. Note also that Hoyt \& Schatten (1998) regard observers with individual correction factors above 2 as poor observers whose observations should be discarded if possible.

\subsection{Testing the hypothesis of the equality of means}

We now make quantitative tests of the null hypothesis that a small sample population (sunspot observations during 17921793 ) is statistically similar to a given reference population. These tests can be found in most handbooks and textbooks on statistical analysis (see e.g., Hudson 1964; Agekyan 1972; Sachs 1972). The size of the sample population is too small to analyze the shape of the distribution function, but we can test the hypothesis of the equality of means of the two populations. We considered three reference populations from the reference period of 1850-1996: the minimum, maximum and mid-declining phase populations including all the daily $R_{\mathrm{g}}$ values in 2-year intervals around sunspot minima, maxima and in the middle of the declining phase, respectively. We applied three different statistical tests for both the daily observations (16 daily measurements in the 1792-1793 sample and about 10000 points in each of the reference populations) and for the monthly averages ( 9 and about 320 points, respectively). The means and standard deviations of the reference populations are given in Table 3 .

First, we applied the Single-Sample Sign test to the null hypothesis. To each point of the sample population $R_{\mathrm{i}}$, a sign "-_" or " + " is assigned depending on whether $R_{\mathrm{i}}$ is smaller or greater than the mean value of the reference population, respectively. Then the number of "+" elements $N^{+}$and "-" elements $N^{-}$is counted and the value of $a$ is calculated:

$a=\frac{\min \left(N^{-}, N^{+}\right)-(n-1) / 2}{\sqrt{n / 2}}$,

where $n$ is the size of the sample population. If the sample population has the same mean as the reference population, the mathematical expectation of $a$ is zero. From the value of $a$ the probability $S_{\mathrm{s}}$ of a false rejection of the null hypothesis is calculated. If $a$ is significantly different (at the level of $\beta=1-S_{\mathrm{s}}$ ) from zero then the null hypothesis of the equality of the two means should be rejected at the significance level $\beta$. Note that the value of $S_{\mathrm{s}}<0.05$ indicates that the two populations have significantly different means (at the significance level of 0.95). This test gives a reliable estimate only if the sample size is significantly larger than 10 elements. Only the daily data set fulfills this requirement, and the calculated values of $S_{\mathrm{s}}$ and $a$ are given in Table 3.

Next we applied the so-called $t$-test which computes $t$ value of Student's statistics:

$$
t=\frac{\bar{x}-\bar{y}}{\sqrt{\sigma_{x}^{2} / n_{x}+\sigma_{y}^{2} / n_{y}}},
$$

where $\bar{x}, \sigma_{x}, n_{x}$ and $\bar{y}, \sigma_{y}, n_{y}$ are the mean, standard deviation and the size of the sample and reference populations, respectively. We adopted here $\bar{x}=16.2, \sigma_{x}=7.6$ and $n_{x}=9$ for the monthly data and $\bar{x}=25, \sigma_{x}=36$ and $n_{x}=16$ for the daily data in 1792-1793. The values of $\bar{y}$ and $\sigma_{y}$ for the reference populations are given in Table 3 . From the $t$-statistics, one can compute the significance level $S_{\mathrm{t}}$ to accept the null hypothesis (or reject it at the level of $1-S_{\mathrm{t}}$ ), with the values of $S_{\mathrm{t}}$ given in Table 3 .

As a third test we applied the non-parametric Wilcoxon Rank Sum test which tests the null hypothesis of the relative unbiasedness of the two populations. There the $z$-statistics is computed

$z=\frac{m_{\mathrm{u}}-U}{\sigma_{\mathrm{u}}}$,

where $U$ is the rank sum of the sample population $x$, and $m_{\mathrm{u}}=\frac{n_{x}\left(n_{x}+n_{y}+1\right)}{2}$ and $\sigma_{\mathrm{u}}=\sqrt{\frac{n_{x} n_{y}\left(n_{x}+n_{y}+1\right)}{12}}$ are the mathematical expectations of the mean and standard deviation of $U$. Then, the value of the probability $S_{\mathrm{W}}$ to accept the null hypothesis is calculated from $z$. The results of this test (see Table 3) imply that the sample population is likely unbiased with respect to the minimum-like reference population but it is significantly biased with respect to both the mid-declining and maximumlike reference populations. 
Table 3. The probability of a false rejection of the null hypothesis that the sample and the reference populations have equal means, according to the Single-Sample Sign test $S_{\mathrm{s}}$, the $t$-test $S_{\mathrm{t}}$ and the Wilcoxon Rank Sum test $S_{\mathrm{W}}$. The corresponding values of $a, t$ and $z$ are given in brackets.

\begin{tabular}{cccc}
\hline \hline & minimum & mid-decl & maximum \\
\hline daily $R_{\mathrm{g}}$ & $10 \pm 12.3$ & $55 \pm 34$ & $115 \pm 48$ \\
$S_{\mathrm{s}}(a)$ & $0.85(0.18)$ & $0.05(-2)$ & $0.03(-2.3)$ \\
$S_{\mathrm{t}}(t)$ & $0.16(1.5)$ & $0.0043(-3.4)$ & $10^{-6}(-8.6)$ \\
$S_{\mathrm{W}}(z)$ & $0.28(-0.58)$ & $3 \cdot 10^{-5}(4)$ & $0(5.7)$ \\
\hline monthly $R_{\mathrm{g}}$ & $10 \pm 8.3$ & $55 \pm 27$ & $115 \pm 39$ \\
$S_{\mathrm{t}}(t)$ & $0.03(2.6)$ & $10^{-10}(-14)$ & $0.0(-27)$ \\
$S_{\mathrm{W}}(z)$ & $0.18(0.88)$ & $10^{-5}(4.2)$ & $0.0(5.7)$ \\
\hline
\end{tabular}

The results of all the above tests are consistent with each other and suggest that only the minimum-like reference population may have the same mean as the 1792-1793 sample, while the hypothesis of the equality of the means should be rejected for both the maximum and the declining phase reference populations at a high significance level. This result is robust and reliable, being confirmed by three different and independent statistical tests and for two different time resolutions (daily vs. monthly). Note that the first and third tests do not require any statistical estimates (e.g., mean, error) of the sample population, and are therefore independent of our analysis of this population presented above. (Moreover, the third test is even independent of the statistical estimates of the reference populations). This implies, contrary to K02, that the sample population of 1792-1793 is statistically similar to the minimum-like reference populations and significantly different from both the maximum and declining phase populations.

\section{Comments to other arguments in $\mathrm{KO2}$}

Here we comment on some indirect arguments raised by K02 against the possibility of the new cycle.

Length of the lost cycle. K02 misinterpreted the dates of cycle extremes suggested in U01. E.g., when defining the length of the lost cycle (see Table 1 of K02), they adopted from U01 only the date of the minimum between cycles $3^{\prime}$ and $4^{\prime}$ (called $4^{*}$ and $4 \mathrm{a}$ in $\mathrm{K} 02$ ). As the date of the next minimum (between cycles 4' (4a) and 5) they used the "official" minimum in 1798.3, leading to a very short length for the lost cycle. However, this official minimum (dotted vertical bar in Fig. 1) was calculated using the Wolf sunspot series, which is different from the group sunspot series analyzed here, in U01 and in K02. Applying the standard 13-month running mean (see, e.g., Gleissberg 1944; Harvey \& White 1999) to the GSN series we found the minimum to be in December 1799 as given in Table 3 in U01. (The 13-month running mean gives the value of 5.4 in 1798.3 and 3.6 in 1799.9). This implies that the length of the lost cycle $4^{\prime}$ is about 7 years (as suggested in U01, U02), and not 5 years as in Table 1 of K02. This modifies the whole analysis of cycle lengths as shown in Figs. 7 and 8 in K02. An appropriate analysis of cycle lengths is given in U02, together with the analysis of the relation between the cycle amplitude and the length of the ascending and descending phases of a cycle (the so-called Waldmeier relations; Waldmeier 1960). The results presented in U02 imply that the introduction of the new cycle does not change significantly the cycle length distribution or the length-vs-amplitude statistics.

Naked-eye observations. As another argument that "this time (i.e., 1792-1793) did not correspond to sunspot minimum", K02 used the fact that a naked-eye sunspot observation was reported in 1792 (Yau \& Stephenson 1988; see also Fig. 1). However, there is another naked-eye observation in Feb. 1799 (Yau \& Stephenson 1988) which was not included in Fig. 1 of K02. This observation falls between the official and the suggested minimum of cycle 5 , in a period which was well covered by sunspot observations and when sunspot activity was even lower than the average level in 1792 (see Fig. 1). Note also that eleven naked-eye sunspot observations are listed in the same catalogue during the Maunder minimum when sunspots were extremely sparse. This implies that, contrary to the suggestion of K02, a naked-eye sunspot observation in 1792 does not exclude the possibility of an additional sunspot minimum in 1792-1793. Moreover, as argued by Eddy (1976, 1983), nakedeye observations alone are not a reliable indicator of sunspot activity.

Skewness. Figure 9 and the end of Sect. 5 of K02 discuss skewness which is supposed to be "a quantitative measure of a cycle profile". This is apparently a lapse since K02 calculated the skewness of the distribution function of $R_{\mathrm{g}}$ values, not of the cycle shape. (Note also that the equation defining skewness in K02 has a typo, missing the power 3). Also, we would like to note that calculating the skewness of sunspot number distribution for the new cycle using only available observations leads to an overestimate because sunspot numbers from the minimum and ascending phases are under-represented in the distribution. If one uses the sunspot activity profile suggested in U01 to fill the gaps in the beginning of the new cycle (see also Fig. 1), the corresponding skewness will be 0.76 , i.e., far below the value of 2.7 given in K02. Accordingly, the value of the skewness of the new cycle is well within the range of other cycles and does not form an exceptional outlier as suggested in K02.

Cosmogenic radionuclides. On the basis of a visual analysis of the cosmogenic ${ }^{10} \mathrm{Be}$ and ${ }^{14} \mathrm{C}$ time series, K02 made the correct conclusion that they do not provide evidence for an extra cycle in 1790s. However, in U02 we have analysed by numerical modeling (using models by Solanki et al. 2000 and Usoskin et al. 2002b) the response of ${ }^{10} \mathrm{Be}$ isotope concentration to the standard sunspot activity in 1790s and the new sunspot activity profile including the new cycle. The results clearly demonstrate that the differences between the two ${ }^{10} \mathrm{Be}$ response profiles are significantly below observational errors. Therefore, the ${ }^{10} \mathrm{Be}$ data are not able to distinguish between the two alternatives. Moreover, the radiocarbon ${ }^{14} \mathrm{C}$ isotope is even less sensitive than ${ }^{10} \mathrm{Be}$ to the fast and rather small changes of solar activity in 1790 s implied by the new cycle. Therefore, as argued in U02, the cosmogenic radionuclide data can neither prove nor disprove the existence of the suggested new cycle in $1790 \mathrm{~s}$.

Aurorae. An analysis of auroral observations in 1790's reveals a small but distinct peak of auroral activity in 1796-1797 (e.g., K02, U02), i.e., a couple of years after the suggested 
additional maximum. Although the existence of this peak which appears in the three independent data series analyzed in K02 and U02 is beyond doubt, its origin can be questioned. In U02 this peak was interpreted as the main peak of auroral activity in cycle $4^{\prime}(4 \mathrm{a})$. This would be in accordance with the common situation where auroral maxima often occur a couple of years after the sunspot cycle maximum. On the other hand, K02 regarded it to be due to the recurrent activity caused by high speed streams occurring very late in cycle $3^{\prime}\left(4^{*}\right)$. We note that the recurrent streams usually occur earlier in the cycle and definitely lead to a much higher peak, which is often higher or of the same order of magnitude as the main peak. Rather, the peak in 1796-1797 was only about $10 \%$ of the main auroral activity peak of cycle $3^{\prime}\left(4^{*}\right)$ and occurred just prior to the official minimum of cycle 4 . Therefore, the existence of the new cycle is not contradicted but slightly favored by the auroral data.

\section{Conclusions}

We have performed a careful statistical analysis of the sunspot observations in 1790s in order to further study the possibility of a lost cycle at this time (Usoskin et al. 2001). Using three independent statistical tests, we have shown that the average level of sunspot activity in 1792-1793 is similar to that around sunspot cycle minima during the more recent, well observed years (1850-1996), but is significantly different from the activity either in the mid-declining phase or around sunspot maxima. This is contrary to the results presented by Krivova et al. (2002) who, when calculating monthly and yearly sunspot number averages, did not take into account the extreme sparseness of sunspot observations and the implied inaccuracy of the calculated monthly and yearly means. Our results show that the existence of a new cycle in 1790s does not contradict with any available sunspot observations or indirect solar proxies (see also U01, U02). Moreover, our refined analysis of sunspot activity in 1790s gives additional evidence for a sunspot minimum in 1792-93, supporting the existence of a new cycle in 1790's.

Acknowledgements. The financial support by the Academy of Finland is gratefully acknowledged. GAK was partly supported by the program "Non-stationary Processes in Astronomy" of Russian Academy of Sciences.

\section{Appendix A: Analysis of measurements with unequal accuracy}

The method of weighted average is a standard approach to analyze a series of measurements with unequal accuracy. Let us assume that the individual data points $R_{\mathrm{i}}$ are independent measurements of the same quantity $\bar{R}$ (e.g., the average sunspot number in 1792-1793) but with individual errors $\sigma_{\mathrm{i}}$. The individual weights $w_{\mathrm{i}}$ are inversely proportional to the squared errors $w_{\mathrm{i}}=1 / \sigma_{\mathrm{i}}^{2}$. The weighted average is then calculated as

$\xi=\frac{1}{w} \sum_{\mathrm{i}} w_{\mathrm{i}} R_{\mathrm{i}}$

where $w=\sum w_{\mathrm{i}}$. The expected mean error of $\xi$ is

$\sigma_{\xi}=1 / \sqrt{w}$.

On the other hand, the actual mean error of $\xi$ can be calculated from

$\sigma_{\xi}^{*}=\sqrt{\frac{1}{(n-1) w} \sum w_{\mathrm{i}}\left(R_{\mathrm{i}}-\xi\right)^{2}}$.

In an ideal case, the values of $\sigma_{\xi}^{*}$ and $\sigma_{\xi}$ should be equal but generally they are not since the individual measurements always contain random errors and may also contain systematic errors. If $\sigma_{\xi}^{*}<\sigma_{\xi}$, the origin of the difference between them is random, and their arithmetic average can be taken as the final estimate of the mean error of $\xi$ :

$\sigma_{\xi}^{f}=\left(\sigma_{\xi}^{*}+\sigma_{\xi}\right) / 2$

If, however, $\sigma_{\xi}^{*}>\sigma_{\xi}$, then

$\sigma_{\xi}^{f}=\sigma_{\xi}^{*}$,

and a factor $l$ should be calculated:

$l=\frac{\left|\sigma_{\xi}^{* 2}-\sigma_{\xi}^{2}\right|}{\sigma_{\xi}^{2}} \cdot \sqrt{\frac{n-1}{2}}$.

If $l<2$ then the difference between $\sigma_{\xi}^{*}$ and $\sigma_{\xi}$ is most likely of random origin (at the significance level of 0.95 ), otherwise a systematic error exists in the measurement series.

Finally one can say that the real value of $\bar{R}$ lies within the interval of $\xi \pm \sigma_{\xi}^{f}$ with the confidence level of 0.68 .

\section{References}

Agekyan, T. A. 1972, The Basics of Errors Theory for Astronomers and Physicists (Moscow: Nauka, in Russian)

Eddy, J. A. 1976, Science, 192, 1189

Eddy, J. A. 1983, Sol. Phys., 89, 195

Gleissberg, W. A. 1944, Terr. Magn. Atm. Electr., 49, 243

Harvey, K. L., \& White, O. R. 1999, J. Geophys. Res., 104, 19759

Hoyt D. V., \& K. Schatten 1998, Sol. Phys., 179, 189

Hudson, D. 1964, Statistics: Lectures on Elementary Statistics and Probability (Geneva)

Krivova, N. A., Solanki, S. K., \& Beer, J. 2002, A\&A, 396, 235

Sachs, L. 1972, Statistische Auswaertungsmethoden (Berlin: Springer-Verlag)

Solanki, S. K., Schüssler, M., \& Fligge, M. 2000, Nature, 408, 445

Usoskin, I. G., Mursula, K., \& Kovaltsov, G. A. 2001, A\&A, 370, L31

Usoskin, I. G., Mursula, K., \& Kovaltsov, G. A. 2002a, Geophys. Res. Lett., 29, 36

Usoskin, I. G., Mursula, K., Solanki, S., Schüssler, M., \& Kovaltsov, G. A. 2002b, J. Geophys. Res., 107, 13

Waldmeier, M. 1960, The Sunspot Activity in the Years 1610-1960 (Zurich: Zurich Schulthess \& Company AG)

Yua, K. K., \& Stephenson, F. R. 1988, Quat. J. R. Astron. Soc., 29, 175 